\title{
Polaron dynamics and bipolaron condensation in cuprates
}

\author{
A. S. Alexandrov \\ Department of Physics, Loughborough University, Loughborough LE11 3TU, United Kingdom
}

(Received 13 January 1999; revised manuscript received 22 November 1999)

\begin{abstract}
Based on the exact cluster diagonalization and recent Quantum Monte Carlo simulations we analyze dynamic properties of small polarons and bipolarons formed by short-range (Holstein) and long-range (Fröhlich) electron-phonon interactions. We show that the exact results agree well with canonical Holstein theory for a cluster and with Lang-Firsov theory for a lattice. Lang-Firsov theory of a single polaron and our $1 / \lambda$ perturbation expansion for a multipolaron system are practically exact in a wide range of the adiabatic parameter $\omega / t$ and the electron-phonon coupling $\lambda$ for a long-range interaction. (Bi)polarons exist in the itinerant Bloch states at temperatures below the characteristic phonon frequency no matter which values the parameters of the system take. We show that recent claims by several authors with regards to a breakdown of Holstein-Lang-Firsov theory of a small polaron and the "impossibility" of bipolaronic superconductivity are the result of an erroneous interpretation of the electronic energy levels of the two-site Holstein model and a misunderstanding of the electron-phonon interaction in ionic solids with polaronic carriers. A "phase" diagram in $t / \omega-\lambda$ space is proposed to elucidate the BCS and (bi)polaronic domains. Bipolaron theory provides a parameter-free expression for the superconducting critical temperature of layered cuprates. Crystallization of the (bi)polaronic liquid is shown to be impossible in the range of the parameters typical for cuprates. The small Fröhlich polaron has spectral features compatible with single-particle tunneling and photoemission in cuprates.
\end{abstract}

\section{INTRODUCTION}

The basic features of small polarons were well recognized a long time ago by Tjablikov ${ }^{1}$ Yamashita and Kurosava, ${ }^{2}$ Sewell, ${ }^{3}$ Holstein, ${ }^{4}$ Lang and Firsov, ${ }^{5}$ Kudinov and Firsov, ${ }^{6}$ and others, and described in several review papers and textbooks. ${ }^{7-13}$ The main feature is the exponential reduction of the bandwidth at intermediate and large values of the electron-phonon coupling $\lambda$, resulting in a coherent small polaron tunneling at low temperatures and a thermally activated hopping at high temperatures. The polaronic bandwidth decreases with increasing temperature. A crossover from the polaronic Bloch states to incoherent hopping takes place at temperatures $T \simeq \omega / 2$ or even higher, where $\omega$ is the characteristic phonon frequency, $\hbar=c=k_{B}=1$. The numerical solution for several vibrating molecules coupled with one or two electrons ${ }^{14}$ revealed an agreement of the numerical bandwidth with the analytical Holstein results at large $\lambda$ both in the nonadiabatic, $\omega \geqslant t$, and adiabatic, $\omega \leqslant t$, regimes $(t$ is the hopping integral). For a multipolaron system a $1 / \lambda$ perturbation theory has been developed ${ }^{15,16}$, which allowed us to extend the BCS theory to the strong-coupling regime $\lambda$ $>1$ and predict the transition to a Bose liquid of $2 e$ charged bipolarons in the crossover region of intermediate values of the BCS coupling constant $\lambda .{ }^{15}$ The renormalized phonon frequencies were obtained ${ }^{17,16}$ in agreement with the numerical results. ${ }^{14}$ The theory has been applied to cuprates ${ }^{18,9,19}$ and more recently to manganites, ${ }^{20}$ providing a description of many unusual properties of these materials ranging from high- $T_{c}$ superconductivity in cuprates to colossal magnetoresistance (CMR) and ferromagnetism in doped manganites. At the same time a few objections ${ }^{21-25}$ have been raised with respect to the Holstein-Lang-Firsov theory of small polarons and the bipolaron theory of superconductivity.

In this paper the polaron dynamics and damping are dis- cussed in more detail to show that small polarons (and bipolarons) exist in the itinerant (Bloch) states at zero temperature no matter which values the parameters of the translationally invariant electron-phonon system take. We analyze the opposite claims, ${ }^{21,22,24}$ elucidating the origin of the controversy. Screening in a multipolaron system is discussed to show that the long-range Fröhlich interaction cannot be reduced to a short-range one, and (bi)polarons exist in a liquid state. The Fröhlich interaction leads to relatively light polarons with the atomic size of the wave function and a large size of the phonon cloud. We suggest the $t / \omega-\lambda$ "phase" diagram with polaronic and bipolaronic domains and show that the effective mass of (bi)polaronic carriers in cuprates fits well the values of their superconducting critical temperature and the London penetration depth. The polaron spectral features are shown to be compatible with the singleparticle tunneling and photoemission in cuprates.

\section{POLARON BAND}

The classical approach to the small-polaron problem is based on the canonical displacement (Lang-Firsov) transformation of the electron-phonon Hamiltonian, ${ }^{5}$ allowing for the summation of all diagrams including the vertex corrections,

$$
\begin{aligned}
H= & \sum_{i, j} t(\mathbf{m}-\mathbf{n}) \delta_{s, s^{\prime}} c_{i}^{\dagger} c_{j}+\sum_{\mathbf{q}, i} \omega_{\mathbf{q}} \hat{n}_{i}\left[u_{i}(\mathbf{q}) d_{\mathbf{q}}+\text { H.c. }\right] \\
& +\sum_{\mathbf{q}} \omega_{\mathbf{q}}\left(d_{\mathbf{q}}^{\dagger} d_{\mathbf{q}}+1 / 2\right),
\end{aligned}
$$

with the bare hopping integral $t(\mathbf{m})$ and the matrix element of the electron-phonon interaction: 


$$
u_{i}(\mathbf{q})=\frac{1}{\sqrt{2 N}} \gamma(\mathbf{q}) e^{i \mathbf{q} \cdot \mathbf{m}}
$$

Here $i=(\mathbf{m}, s), j=\left(\mathbf{n}, s^{\prime}\right), \hat{n}_{i}=c_{i}^{\dagger} c_{i}$, and $c_{i}, d_{q}$ are the electron (hole) and phonon operators, respectively, and $N$ is the number of sites.

As long as $\lambda>1$, the kinetic energy remains smaller than the interaction energy and a self-consistent treatment of the many-electron system strongly coupled with phonons is possible with the " $1 / \lambda$ " expansion technique. ${ }^{16}$ This possibility stems from the fact, known for a long time, that there is an exact solution for a single electron in the strong-coupling limit $\lambda \rightarrow \infty$. Following Lang and Firsov, ${ }^{5}$ one can apply the canonical transformation $e^{S}$ to diagonalize the Hamiltonian. The diagonalization is exact if $t(\mathbf{m})=0($ or $\lambda=\infty)$ :

$$
\widetilde{H}=e^{S} H e^{-S},
$$

where

$$
S=\sum_{\mathbf{q}, i} \hat{n}_{i}\left[u_{i}(\mathbf{q}) d_{q}-\text { H.c. }\right] .
$$

The electron operator transforms as

$$
\widetilde{c_{i}}=c_{i} \exp \left(-\sum_{\mathbf{q}} u_{i}(\mathbf{q}) d_{\mathbf{q}}-\text { H.c. }\right)
$$

and the phonon one as

$$
\tilde{d}_{\mathbf{q}}=d_{\mathbf{q}}+\sum_{i} \hat{n}_{i} u_{i}^{*}(\mathbf{q})
$$

It follows from Eq. (6) that the Lang-Firsov canonical transformation shifts ions to new equilibrium positions. In a more general sense it changes the boson vacuum. As a result,

$$
\begin{aligned}
\widetilde{H}= & \sum_{i, j} \hat{\sigma}_{i j} c_{i}^{\dagger} c_{j}-E_{p} \sum_{i} \hat{n}_{i}+\sum_{\mathbf{q}} \omega_{\mathbf{q}}\left(d_{\mathbf{q}}^{\dagger} d_{\mathbf{q}}+1 / 2\right) \\
& +\frac{1}{2} \sum_{i \neq j} v_{i j} \hat{n}_{i} \hat{n}_{j},
\end{aligned}
$$

where

$$
\hat{\sigma}_{i j}=t(\mathbf{m}-\mathbf{n}) \delta_{s, s^{\prime}} \exp \left(\sum_{\mathbf{q}}\left[u_{i}(\mathbf{q})-u_{j}(\mathbf{q})\right] d_{\mathbf{q}}-\text { H.c. }\right)
$$

is a renormalized hopping integral depending on the phonon variables and

$$
v_{i j}=-\frac{1}{N} \sum_{\mathbf{q}}|\gamma(\mathbf{q})|^{2} \omega_{\mathbf{q}} \cos [\mathbf{q} \cdot(\mathbf{m}-\mathbf{n})]
$$

is the the attractive interaction of polarons owing to the local lattice deformation.

In a strong-coupling limit $\lambda \rightarrow \infty$, one can neglect the hopping term of the transformed Hamiltonian. The rest has analytically determined eigenstates and eigenvalues. The eigenstates $|\tilde{N}\rangle=\left|n_{i}, n_{\mathbf{q}}\right\rangle$ are classified with the polaron $n_{\mathbf{m}, s}$ and phonon $n_{\mathbf{q}}$ occupation numbers, and the energy levels are

$$
E=-E_{p} \sum_{i} n_{i}+\frac{1}{2} \sum_{i \neq j} v_{i j} n_{i} n_{j}+\sum_{\mathbf{q}} \omega_{\mathbf{q}}\left(n_{\mathbf{q}}+1 / 2\right),
$$

with $n_{i}=0,1$ and $n_{\mathbf{q}}=0,1,2,3, \ldots, \infty$.

Hence, the Hamiltonian, Eq. (7), in zero order with respect to the hopping describes localized polarons and independent phonons which are vibrations of ions relative to new equilibrium positions depending on the polaron occupation numbers. The phonon frequencies remain unchanged in this limit. The middle of the electronic band falls by the polaronic level shift $E_{p}$ as a result of a potential well created by the lattice deformation:

$$
E_{p}=\frac{1}{2 N} \sum_{\mathbf{q}}|\gamma(\mathbf{q})|^{2} \omega_{\mathbf{q}} .
$$

First, we limit our discussion to a single-polaron problem with no polaron-polaron interaction. The effects of the interaction (including also the direct Coulomb repulsion) such as the bipolaron formation and screening are discussed in the final sections of this paper.

With the finite hopping term polarons tunnel in a narrow band owing to the degeneracy of the zero-order Hamiltonian with respect to the site position of a single polaron in a regular lattice. To see it one can apply perturbation theory using $1 / \lambda$ as a small parameter with $\lambda \equiv E_{p} / z t$ ( $z$ is the coordination lattice number and $t$ the nearest-neighbor hopping integral). The proper (Bloch) set of $N$ degenerate zero-order eigenstates of the lowest-energy level $\left(-E_{p}\right)$ of the unperturbed Hamiltonian is

$$
|\mathbf{k}, 0\rangle=\frac{1}{\sqrt{N}} \sum_{\mathbf{m}} c_{\mathbf{m}}^{\dagger} \exp (i \mathbf{k} \cdot \mathbf{m})|0\rangle,
$$

where $|0\rangle$ is the vacuum. By applying textbook perturbation theory, one readily calculates the lowest-energy levels of the polaron in a crystal. Up to second order in the hopping integral, the result is

$$
E(\mathbf{k})=-E_{p}+\epsilon_{\mathbf{k}}-\sum_{\mathbf{k}^{\prime}, n} \frac{\left.\mid\left\langle\mathbf{k}, 0\left|\sum_{i, j} \hat{\sigma}_{i, j} c_{i}^{\dagger} c_{j}\right| \mathbf{k}^{\prime}, n_{\mathbf{q}}\right\rangle\right)\left.\right|^{2}}{\sum_{\mathbf{q}} \omega_{\mathbf{q}} n_{\mathbf{q}}},
$$

with $\left|\mathbf{k}^{\prime}, n_{\mathbf{q}}\right\rangle$ the exited states of the unperturbed Hamiltonian with one electron and at least one real phonon. The second term in Eqs. (13), which is linear with respect to the bare hopping $t$, determines the small polaron band dispersion as

$$
\epsilon_{\mathbf{k}}=\sum_{\mathbf{m}} t(\mathbf{m}) e^{-g^{2}(\mathbf{m})} \exp (-i \mathbf{k} \cdot \mathbf{m})
$$

with the band-narrowing factor (at zero temperature)

$$
g^{2}(\mathbf{m})=\frac{1}{2 N} \sum_{\mathbf{q}}|\gamma(\mathbf{q})|^{2}[1-\cos (\mathbf{q} \cdot \mathbf{m})] .
$$

The third term in Eq. (13), quadratic in $t$, yields a negative k-independent correction to the polaron level shift of the order of $1 / \lambda^{2}$. The origin of this correction, which is much 


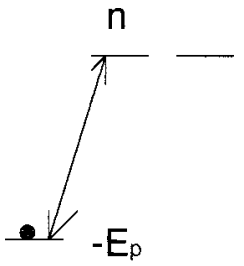

$\mathrm{m}$

FIG. 1. "Back-forth" virtual transitions of the polaron without any transfer of the lattice deformation from the site $\mathbf{m}$ to the neighboring site $\mathbf{n}$. These transitions shift the middle of the polaron band, but they do not produce any real charge delocalization.

larger than the first order in $t$ contribution (containing a small exponent), is understood from Fig. 1. The polaron localized in the potential well of the depth $E_{p}$ on the site $\mathbf{m}$ hops onto a neighboring site $\mathbf{n}$ with no deformation around and comes back. As any second order correction, this transition shifts the energy down by an amount $\sim-t^{2} / E_{p}$. It has little to do with the polaron effective mass and the polaron tunneling mobility because the lattice deformation around $\mathbf{m}$ does not follow the electron. The electron hops "back and forth", many times (about $e^{g^{2}}$ ) "waiting", for a sufficient lattice deformation to appear around the site n. Only after it "creates" the deformation around $\mathbf{n}$, the electron tunnels onto the next site together with the deformation.

\section{DAMPING OF THE POLARON BAND}

The polaron band is exponentially narrow, Eq. (14). Hence one can raise a question concerning its existence in real solids. At zero temperature the perturbation term of the transformed Hamiltonian conserves momentum because all off-diagonal matrix elements vanish,

$$
\left\langle\mathbf{k}, 0\left|\sum_{i, j} \hat{\sigma}_{i, j} c_{i}^{\dagger} c_{j}\right| \mathbf{k}^{\prime}, 0\right\rangle=0,
$$

if $\mathbf{k} \neq \mathbf{k}^{\prime}$. The absorption or emission of a single highfrequency phonon is forbidden by energy conservation because the polaron half-bandwidth $w \leqslant \omega$. Hence there is no damping of the polaron band at $T=0$ no matter how strong the interaction $\lambda$ and how small the adiabatic ratio $\omega / t$ are. However, the polaron bandwidth depends on temperature. For high temperatures $T \gg \omega / 2$ the band shrinks exponentially with increasing temperature, ${ }^{7,8,13}$

$$
w \simeq z t \exp \left(-\frac{2 E_{p} T}{\omega^{2}}\right) .
$$

On the other hand, the scattering of polarons within their narrow band becomes more important with increasing tem-

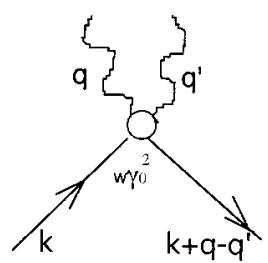

FIG. 2. Two-phonon incoherent scattering responsible for a damping of polaron Bloch states at finite temperatures. perature owing to the simultaneous emission and absorption of phonons, Fig. 2. These incoherent events tend to destroy the coherent polaron tunneling within the band. The corresponding scattering rate is given by the Fermi golden rule as

$$
\begin{aligned}
\frac{1}{\tau}= & 2 \pi\left\langle\sum_{\mathbf{q}, \mathbf{q}^{\prime}}\right|\left\langle\mathbf{k}+\mathbf{q}-\mathbf{q}^{\prime}, n_{\mathbf{q}}-1, n_{\mathbf{q}^{\prime}}\right. \\
& \left.\left.+1\left|\sum_{i, j} \hat{\sigma}_{i, j} c_{i}^{\dagger} c_{j}\right| \mathbf{k}, n_{\mathbf{q}}, n_{\mathbf{q}^{\prime}}\right\rangle\left.\right|^{2} \delta\left(\boldsymbol{\epsilon}_{\mathbf{k}}-\epsilon_{\mathbf{k}+\mathbf{q}^{-}-\mathbf{q}^{\prime}}\right)\right\rangle .
\end{aligned}
$$

Expanding $\hat{\sigma}_{i j}$ operators in the powers of the phonon creation and annihilation operators, one estimates the matrix element of the two-phonon scattering as

$$
\begin{aligned}
& \left|\left\langle\mathbf{k}+\mathbf{q}-\mathbf{q}^{\prime}, n_{\mathbf{q}}-1, n_{\mathbf{q}^{\prime}}+1|| \sum_{i, j} \hat{\sigma}_{i, j} c_{i}^{\dagger} c_{j} \mid \mathbf{k}, n_{\mathbf{q}}, n_{\mathbf{q}^{\prime}}\right\rangle\right| \\
& \sim \frac{1}{N} w \gamma_{0}^{2} \sqrt{n_{\mathbf{q}}} \sqrt{n_{\mathbf{q}^{\prime}}+1} .
\end{aligned}
$$

Substituting this estimate into Eq. (18) and using the definition of the density of states in the polaron band,

$$
N_{p}(\xi) \equiv \frac{1}{N} \sum_{\mathbf{k}} \delta\left(\xi-\epsilon_{\mathbf{k}}\right) \simeq \frac{1}{2 w},
$$

one obtains

$$
\frac{1}{\tau} \simeq w \gamma_{0}^{4} n_{\omega}\left(1+n_{\omega}\right)
$$

with the momentum-independent $\gamma(\mathbf{q})=\gamma_{0}$ and the phonon distribution function $n_{\omega}=[\exp (\omega / T)-1]^{-1}$. The polaron band is well defined if

$$
\frac{1}{\tau}<w
$$

which is satisfied for a wide temperature range

$$
T \leqslant \frac{\omega}{\ln \gamma_{0}^{4}}
$$

below about half of the characteristic phonon frequency for the relevant values of $\gamma_{0}^{2}$. The incoherent thermally activated hopping dominates in the polaron motion at higher temperatures where the polaronic states cannot be classified by their momenta.

\section{TWO-SITE HOLSTEIN MODEL: EXACT VERSUS ANALYTICAL SOLUTION}

The major polaronic features described above have been known for a long time starting from the pioneering works by Tjablikov, ${ }^{1}$ Holstein, ${ }^{4}$ and Lang and Firsov (LF). ${ }^{5}$ During the 

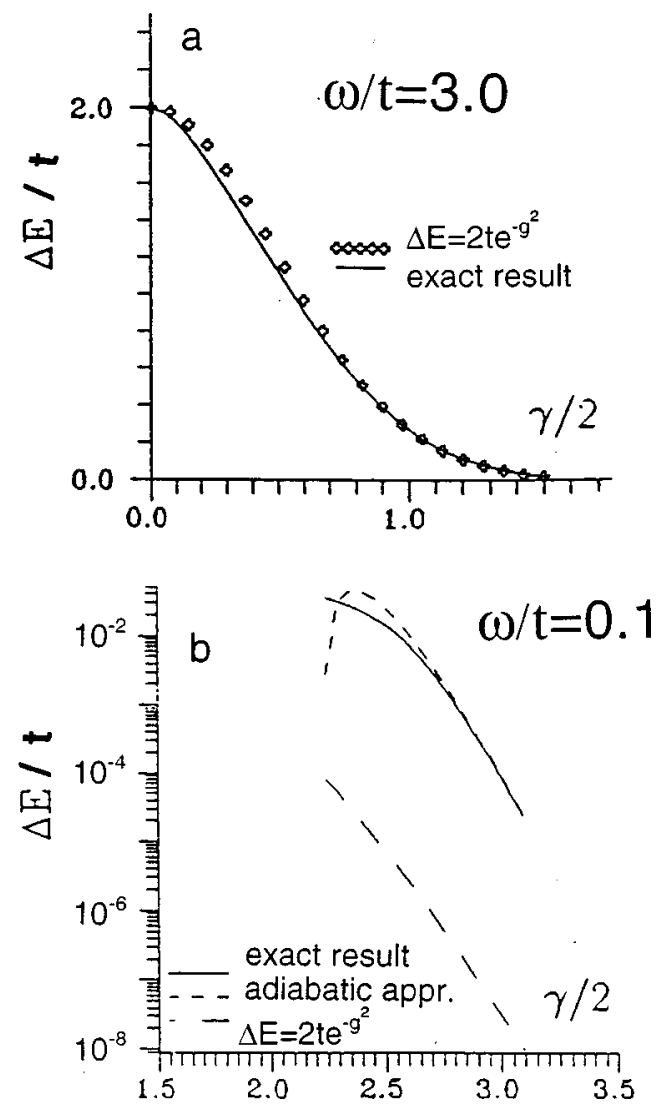

FIG. 3. Exact (cluster diagonalization) "bandwidths" of the two-site Holstein model compared with the analytical nonadiabatic, Eq. (14), and adiabatic, Eq. (24), bandwidths.

last decade, the small polaron problem has gained further attention as relevant to high- $T_{c}$ and other oxides (for recent publications, see Refs. 13 and 26-38 and references therein). The efforts were mainly directed towards extension of the theory to the intermediate region of the coupling, $\lambda \sim 1$, and the adiabatic ratio $\omega / t \sim 1$, and a study of the polaron-polaron correlations. It has been shown ${ }^{39,16}$ that the expansion parameter is actually $1 / 2 z \lambda^{2}$, so the analytical perturbation theory has a wider region of applicability than one can expect using simple physical arguments. However, it is not clear how fast the expansion converges. While the ground-state energy (about $-E_{p}$ ) is not very sensitive to the parameters, the effective mass and bandwidth strongly depend on the polaron size and adiabatic ratio.

The most reliable results for the intermediate region have been obtained with the exact numerical diagonalization of vibrating clusters ${ }^{40,14,41,29}$ and quantum Monte Carlo simulations. ${ }^{42,37,43}$ Numerical diagonalization of the two-site one-electron Holstein model in the adiabatic $\omega / t<1$ and in the nonadiabatic $\omega / t>1$ regimes shows that perturbation theory is almost exact in the nonadiabatic regime for all values of the coupling constant, Fig. 3(a). There is no agreement in the adiabatic region, where the first-order perturbation expression overestimates the polaron mass by a few orders of magnitude. A much lower effective mass of the adiabatic small polaron in the intermediate-coupling region compared with that estimated by the first-order perturbation theory is revealed in Fig. 3(b). A poor convergence of the perturbation expansion is explained by the appearance of the familiar double-well potential ${ }^{4}$ in the adiabatic limit. The tunneling probability is extremely sensitive to the shape of this potential. The splitting of levels for the two-site cluster is well described by the Holstein quasiclassical formula generalized for the intermediate coupling in Ref. 14:

$$
\Delta E=\left(\frac{16 E_{p} \omega}{\pi}\right)^{1 / 2} \beta^{5 / 2} \lambda^{1-\beta}[2(1+\beta)]^{-\beta} e^{-\tilde{g}^{2}},
$$

where $\widetilde{g}^{2}=g^{2}\left(\beta-\{\ln [2 \lambda(1+\beta)]\} / 4 \lambda^{2}\right)$. This generalization takes into account the phonon frequency renormalization $\beta$ $\equiv \widetilde{\omega} / \omega=\left(1-1 / 4 \lambda^{2}\right)^{1 / 2}$ (Ref. 17) and the anharmonic corrections of the order of $1 / \lambda^{2}$ to the turning points. While the small Holstein polaron is only a few times heavier than the bare (unrenormalized) electron in a wide range of coupling for a moderate adiabatic ratio $\omega / t \sim 1$, Fig. 3(a), it becomes quite heavy in the adiabatic regime and for the strong coupling, Fig. 3(b). Thus the numerical results confirm all major polaronic features well understood by Holstein ${ }^{4}$ and others both in the nonadiabatic and adiabatic regimes.

However, analyzing the same numerical problem, de Mello and Ranninger ${ }^{21}$ have recently arrived at the opposite conclusion that "the LF approach, which is generally believed to become exact in the limit of antiadiabaticity and an electron-phonon coupling going to infinity, actually diverges (the) most from the exact results precisely in this limit ...." These authors have not provided any physical explanation for their disagreement with all earlier results starting from the pioneering work by Holstein and including the kinetic theory of strongly coupled electron-phonon systems. ${ }^{8}$ It has become clear that the conclusion of Ref. 21 is an artifact of an erroneous identification of the polaron kinetic energy. ${ }^{26,44}$ de Mello and Ranninger ${ }^{45}$ subsequently claimed that their definition of the polaron kinetic energy should be attributed to Holstein rather than to themselves and that their interpretation of the dynamic correlation functions of the Holstein model remains valid. We disagree with these claims.

Holstein ${ }^{4}$ distinguished perfectly well the nonadiabatic and adiabatic small polarons as well as the tunneling probability and the corrections to the ground-state energy due to the "back-forth" virtual transitions. The polaronic correlation functions were well established later on in the framework of the theory of optical conductivity. ${ }^{8}$ Recently, Firsov and Kudinov ${ }^{26}$ developed an analytical approach to the twosite model by the use of the expansion technique, which provides the electronic and vibronic terms as well as the wave functions and all correlation functions in any order of powers of $t$. They have found the exponential reduction factor in all orders of the $1 / \lambda$ perturbation expansion, in agreement with the canonical result, Eq. (14). On the other hand, the corrections to the atomic level were found to be as small as $1 / \lambda^{2}$ rather than exponential.

The fundamental error of Ref. 21 originates from a failure to apply properly perturbation theory and to notice the different origins of two terms in Eq. (13). As a measure of the kinetic polaron energy, the authors of Ref. 21 take the correlation function

$$
t_{\mathrm{eff}}=\left\langle-t\left(c_{1}^{\dagger} c_{2}+c_{2}^{\dagger} c_{1}\right)\right\rangle,
$$

where $c_{1,2}$ are annihilation operators on the "left" and "right" molecule (site). Up to second order in $t$, one obtains 


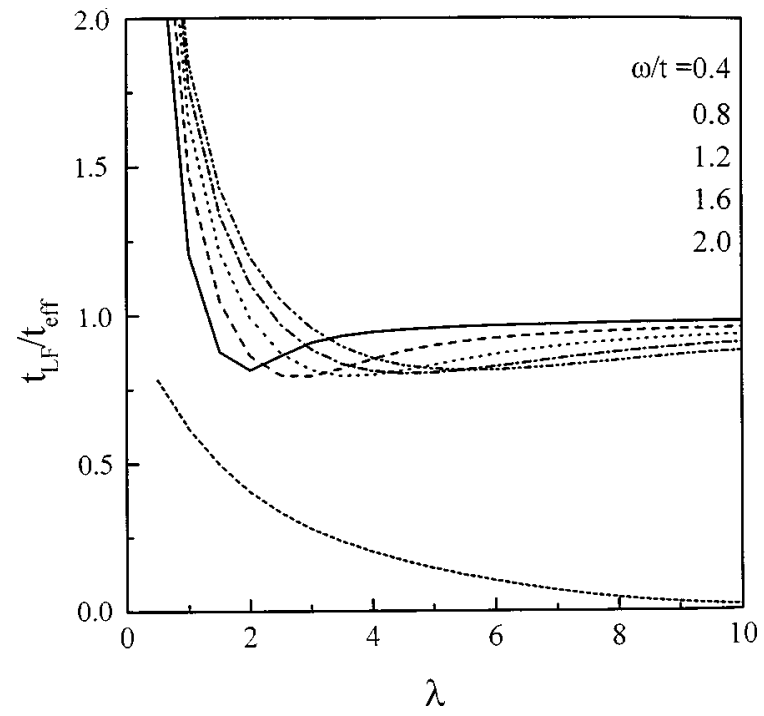

FIG. 4. The ratio of the perturbatively calculated correlator $t_{\mathrm{LF}}$ including the second-order term $\left(\sim 1 / \lambda^{2}\right)$ to the exact one (Ref. 46 ), $t_{\text {eff }}$, for different values of the adiabatic ratio $\omega / t$. The lowest curve represents an incorrect result of Ref. 21 with missing second-order term.

$$
t_{\mathrm{eff}} \simeq t_{\mathrm{LF}} \equiv-t \exp \left(-\frac{\lambda t}{\omega}\right)-\frac{t}{\lambda},
$$

with $\lambda \equiv 2 E_{p} / t$. Only the first exponential term in Eq. (26) corresponds to the polaron kinetic energy, while the second describes the corrections to the middle of the "band" owing to the virtual transitions to the neighboring site as described in Sec. II. Comparing the analytical expression, Eq. (26), with the numerically calculated $t_{\text {eff }}$, one confirms ${ }^{46}$ that the Holstein-Lang-Firsov approach is asymptotically exact, contrary to Ref. 21 . The ratio of this correlation function to the exact one is unity in the large- $\lambda$ limit, Fig. 4, in both the nonadiabatic and adiabatic regimes. The theory of Ref. 21 does not recognize that the main contribution to $t_{\text {eff }}$ comes from the k-independent second-order lowering of the polaron level. The numerically calculated value of $t_{\text {eff }}$ was compared with the first exponentially small term in Eq. (26) alone, ignoring the dominating second term (see the lower curve in Fig. 4). Actually, $t_{\text {eff }}$ does not represent the polaron kinetic energy at all. It includes a large contribution from the virtual "back-forth" transitions to the neighboring site, Fig. 1, which have nothing to do with any real charge delocalization. The misinterpretation of this term led to an incorrect interpretation of the dynamic properties of polarons ${ }^{21}$ and bipolarons ${ }^{22}$ including their correlation functions and damping.

In particular, it was claimed ${ }^{21}$ that "the exact result for the occupation number $n(k)=\left\langle c_{k}^{\dagger} c_{k}\right\rangle$ differs from that of the LF approach qualitatively, and the dynamical coherence of the polaron increases with increasing temperature... so they expect for an infinite lattice a mobility which increases with increasing temperature, while the opposite behavior is found in the classical works ${ }^{4,5}$ on that issue and being based on the LF $1 / \lambda$ perturbative approach.' In arriving at these conclusions the authors did not take into account $1 / \lambda$ corrections to the occupation numbers. By including the first-order correction, we obtain for the two-site model

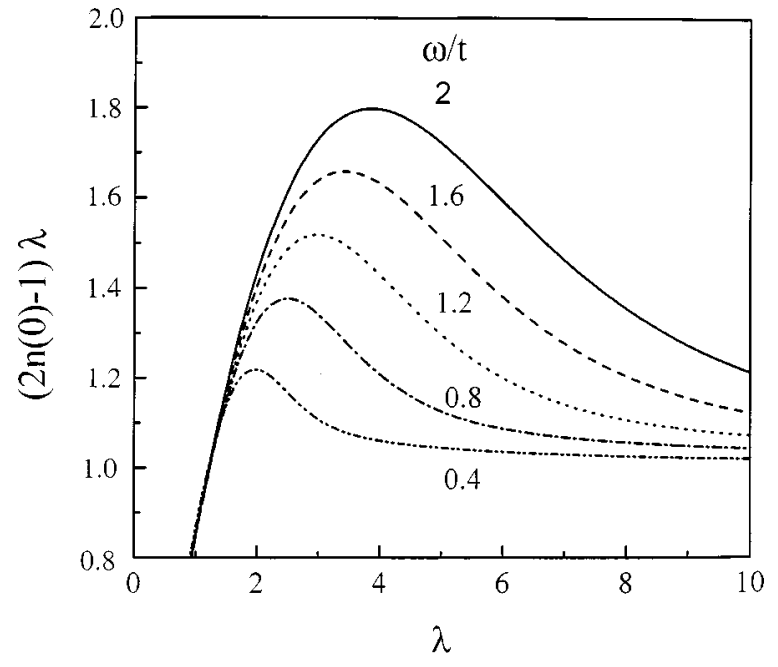

FIG. 5. The exact polaron occupation number (Ref. 46) as a function of $\lambda$ for different values of the adiabatic ratio $\omega / t$. It approaches $\frac{1}{2}(1+1 / \lambda)$ for large $\lambda$, in agreement with Holstein-LangFirsov theory, Eqs. (27).

$$
\begin{aligned}
& n(k=0)=\frac{1}{2}\left[1+\exp \left(-\frac{\lambda t}{\omega}\right)\right]+\frac{1}{2 \lambda}, \\
& n(k=\pi)=\frac{1}{2}\left[1-\exp \left(-\frac{\lambda t}{\omega}\right)\right]-\frac{1}{2 \lambda} .
\end{aligned}
$$

The function $2 \lambda[n(0)-1 / 2]=2 \lambda[1 / 2-n(\pi)]$, numerically calculated by Kabanov, ${ }^{46}$ is shown in Fig. 5. It goes to unity at a large $\lambda$ for any value of adiabaticity $\omega / t$ in agreement with Eqs. (27) and (28). It should be pointed out that a deviation from 1 in Fig. 5 is due to the exponential term in Eq. (27), so that $2 \lambda[n(0)-1 / 2]-1=\lambda \exp (-\lambda t / \omega)$. This exponent is smaller in the adiabatic case $(\omega<t)$ than in the nonadiabatic one $(\omega>t)$ for a fixed value of $\lambda$, which explains why in Fig. 5 the results in the adiabatic regime converge more rapidly to 1 than the results for large $\omega / t$. Hence the electronic occupation numbers for the ground state of the two-site model are in excellent quantitative agreement with Holstein-Lang-Firsov theory contrary to the conclusion of Ref. 21. We also notice that the temperature dependence of $t_{\text {eff }}$ should not be identified with that of the mobility because $t_{\text {eff }}$ has little to do with the tunneling under the deformation barrier and with the polaron kinetics.

Reference 21 also concluded that "the dynamical behavior of the polaronic charge carriers alternate between selftrapped polarons and almost free-carrier behavior, and, in general effects of dynamical delocalization of the electron cannot be obtained by perturbative expansions in terms of $1 / \lambda$ around the LF-approximated oscillator wave function, even in the extreme antiadiabatic limit.' On the contrary, our Fourier analysis ${ }^{44}$ of the numerically calculated timedependent correlation function for the charge fluctuations $\chi_{n n}$ and molecular deformations $\chi_{x x}$ revealed a Fourier component corresponding to coherent polaron tunneling. Its frequency $\nu$ agrees well with that predicted by Holstein theory, $\nu=2 t \exp (-\lambda t / \omega)$. The frequency is found in both $\chi_{n n}$ and $\chi_{x x}$ correlation functions as expected for the tunneling of the electron accompanied by lattice deformation. The Fourier components of $\chi_{n n}$ have a well-defined maximum in the 
high-frequency region. The maximum corresponds to twice the polaronic level shift as it should be for the spectral function of small polarons. ${ }^{13}$

The two-site model does not allow for a self-consistent analysis of the polaron damping, conductivity, or photoemission. They strongly depend on the phonon dispersion and relaxation which are beyond the two-site quantum mechanical problem. Within this model one can easily mistake virtual "back-forth" transitions, Fig. 1, for real charge fluctuations. In Ref. 22 this mistake led to the conclusion that "the residual interaction with the lattice deformations surrounding (bi)polarons leads to a dynamical dephasing between the charge carriers and the local lattice deformations surrounding them and hence destroys any itinerant quasiparticle features." As we have discussed in Sec. III, there is no damping (or dephasing) of small (bi)polarons at low temperatures no matter what the parameters of the system are. Other independent variational and cluster diagonalization studies ${ }^{33,34}$ confirmed that "by the use of the Holstein approximation and the canonical Lang-Firsov approach with appropriate corrections, one obtains an excellent estimate of the coherent bandwidth in both adiabatic and non-adiabatic regimes.', 33

\section{SMALL HOLSTEIN POLARON AND "SMALL FRÖHLICH POLARON",}

The analytical $1 / \lambda$ expansion allows us to analyze both a small Holstein polaron (SHP) with a short-range interaction and a lighter small polaron with a long-range Fröhlich interaction, i.e., mobile small Fröhlich polaron (SFP). ${ }^{47,43}$ To illustrate this point we express the electron-phonon interaction in terms of real displacements $\xi_{\mathbf{n}}$ as ${ }^{43}$

$$
H_{e-\mathrm{ph}}=-\sum_{\mathbf{n}, i} f(\mathbf{m}-\mathbf{n}) \xi_{\mathbf{n}} \hat{n}_{i} .
$$

Here $\quad \xi_{\mathbf{n}}=\Sigma_{\mathbf{q}}\left(2 N M \omega_{\mathbf{q}}\right)^{-1 / 2} \exp (i \mathbf{q} \cdot \mathbf{n}) d_{\mathbf{q}}^{\dagger}+$ H.c. is a normal coordinate at site $\mathbf{n}$, and $f(\mathbf{m}-\mathbf{n})$ $=N^{-1} \Sigma_{\mathbf{q}} \gamma(\mathbf{q})\left(M \omega_{\mathbf{q}}^{3}\right)^{1 / 2} \exp [i \mathbf{q} \cdot(\mathbf{n}-\mathbf{m})]$ is the force between the electron at site $\mathbf{m}$ and the normal coordinate $\xi_{\mathbf{n}}$.

In general, there is no simple relation between the polaronic shift $E_{p}$ and the exponent $g^{2}$ of the mass enhancement. This relation depends on the form of the electronphonon interaction. Indeed, for dispersionless phonons $\omega_{\mathbf{q}}$ $=\omega$, one obtains

$$
E_{p}=\frac{1}{2 M \omega^{2}} \sum_{\mathbf{m}} f^{2}(\mathbf{m}),
$$

while

$$
g^{2}=\frac{1}{2 M \omega^{3}} \sum_{\mathbf{m}}\left[f^{2}(\mathbf{m})-f(\mathbf{m}) f(\mathbf{m}+\mathbf{a})\right],
$$

where $\mathbf{a}$ is the lattice vector. The effective mass renormalization is $m^{*} / m=e^{g^{2}}$, where $m$ is the bare band mass and $1 / m^{*}=\partial^{2} E(\mathbf{k}) / \partial k^{2}$ with $k \rightarrow 0$. If the interaction is local, $f(\mathbf{m})=\kappa \delta_{\mathbf{m}, 0}($ Holstein model $)$, then $g^{2}=E_{p} / \omega$. In general, one has $g^{2}=\gamma E_{p} / \omega$ with a numerical coefficient $\gamma=1$ $-\Sigma_{\mathbf{m}} f(\mathbf{m}) f(\mathbf{m}+\mathbf{a}) / \Sigma_{\mathbf{n}} f^{2}(\mathbf{n})$ less than unity. ${ }^{47}$ Here $M$ is the ion mass.

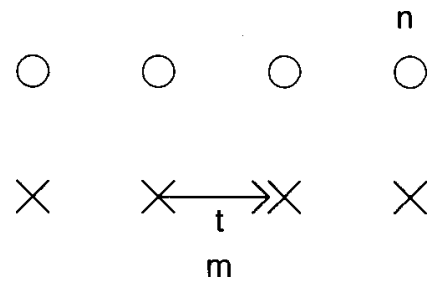

FIG. 6. One-dimensional model of the small Fröhlich polaron on the chain $\times$ interacting with all ions of another chain $\bigcirc$ (Ref. 43).

To calculate $\gamma$ one can introduce a one-dimensional lattice model with a long-range Coulomb interaction between the electron and ions, Fig. $6{ }^{43}$ The electron in a Wannier state on a site $\mathbf{m}$ of the infinite chain $(X)$ interacts with the vibrations of all ions of another chain $(\bigcirc)$ polarized in the direction perpendicular to the chains. The corresponding force is given by

$$
f(\mathbf{m}-\mathbf{n})=\frac{\kappa}{\left(|\mathbf{m}-\mathbf{n}|^{2}+1\right)^{3 / 2}} .
$$

The distance along the chains $|\mathbf{m}-\mathbf{n}|$ is measured in units of the lattice constant $a$; the interchain distance is also $a$. Here and further on we take $a=1$. For this long-range interaction one obtains $E_{p}=1.27 \kappa^{2} /\left(2 M \omega^{2}\right), g^{2}=0.49 \kappa^{2} /\left(2 M \omega^{3}\right)$, and $g^{2}=0.39 E_{p} / \omega$. The effective mass renormalization is much smaller than in the dispersionless Holstein model, roughly as $m_{\mathrm{SFP}}^{*} \propto\left(m_{\mathrm{SHP}}^{*}\right)^{1 / 2}$.

Not only does the small polaron mass strongly depend on the radius of the electron-phonon interaction, but also the range of applicability of the analytical Lang-Firsov theory. While in the case of a short-range (Holstein) interaction this approach is applied only if $\omega \geqslant t$ and $\lambda \gg 1$, the theory appears almost exact in a substantially wider region of parameters for the Fröhlich interaction. The polaron mass in a wide region of the adiabatic parameter and coupling has been recently calculated $^{43}$ with the continuous-time path-integral quantum Monte Carlo (QMC) algorithm, developed by Kornilovich. ${ }^{37}$ This method is free from any systematic finite-size, finite-time-step, and finite-temperature errors and allows for an exact (in the QMC sense) calculation of the ground-state energy and the effective mass of the lattice polaron for any electron-phonon interaction.

At large $\lambda(>1.5)$ we found the SFP to be much lighter than the SHP, while the large Fröhlich polaron (i.e., at $\lambda$ $<1$ ) is heavier than the large Holstein polaron with the same binding energy, Fig. 7. The mass ratio $m_{\mathrm{FP}}^{*} / m_{\mathrm{HP}}^{*}$ is a nonmonotonic function of $\lambda$. The effective mass of small and large Fröhlich polarons, $m_{\mathrm{FP}}^{*}(\lambda)$, is well fitted by a single exponent, which is $e^{0.73 \lambda}$ for $\omega=t$ and $e^{1.4 \lambda}$ for $\omega=0.5 t$, which is not the case for the Holstein polaron (see Sec. IV). The exponents are remarkably close to those obtained with the Lang-Firsov transformation, $e^{0.78 \lambda}$ and $e^{1.56 \lambda}$, respectively. Hence, in the case of the Fröhlich interaction, the transformation is perfectly accurate even in the adiabatic regime $\omega / t \leqslant 1$ for any coupling strength.

Another interesting point is that the size of the SFP and the length over which the distortion spreads are different. In the extreme strong-coupling limit, the Lang-Firsov transformation is exact, and the polaron is entirely localized on one site $\mathbf{m}$. Hence the size of its wave function is the atomic size. On the other hand, the ion displacements, proportional to the 


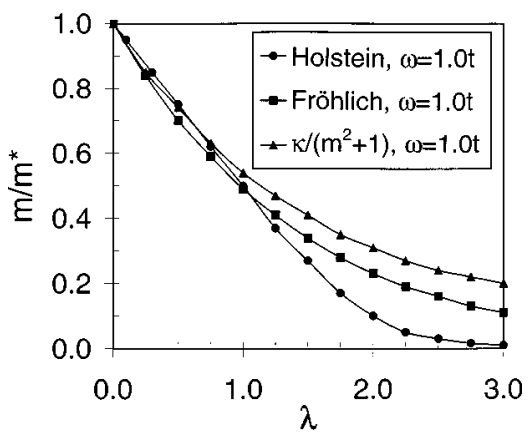

FIG. 7. The ratio of the band mass to the polaron mass as a function of the coupling constant. Polarons become lighter with increasing radius of the electron-phonon interaction, as shown with triangles.

displacement force $f(\mathbf{m}-\mathbf{n})$, spread over a large distance. Their amplitude at a site $\mathbf{n}$ falls with the distance as $1 / \mid \mathbf{m}$ $-\left.\mathbf{n}\right|^{3}$ in our one-dimensional model. The polaron cloud (i.e., lattice distortion) can be more extended than the polaron itself (see, also, Refs. 2, 50, and 47). Such a polaron tunnels with a larger probability than the nondispersive Holstein polaron due to a smaller relative lattice distortion around two neighboring sites. It can be equally called a "large discrete strong-coupling Fröhlich" polaron, if the lattice distortion is included in the definition of its size. On the other hand, historically one referes to a "small" polaron, as a quasiparticle well described by the $1 / \lambda$ expansion technique. With this definition polarons on a lattice are small for any value of the long-range electron-phonon interaction.

The model, Eq. (32), contains only one phonon mode polarized along the $c$ axis, so that the $c$ component of the field from a $c$-polarized dipole falls off with distance as $1 / r^{3}$. An isotropic Fröhlich interaction might be longer ranged than ours, giving rise to a $1 / r^{2}$ law. Consequently, it should yield even lighter polaron mass. This is confirmed numerically ${ }^{51}$ as shown in Fig. 7 for the one-dimensional model with the force $f(\mathbf{m})=\kappa /\left(m^{2}+1\right)$. The fact that the Lang-Firsov transformation is perfectly accurate for the long-range interaction in a wide region of the parameters allows us to generalize this result. Including all phonon polarizations in a three-dimensional lattice, we obtain $m_{\mathrm{SFP}}^{*} \sim\left(m_{\mathrm{SHP}}^{*}\right)^{\gamma}$, with the constant $\gamma=\Sigma_{\mathbf{q}} \gamma^{2}(\mathbf{q})[1-\cos (\mathbf{q} \cdot \mathbf{m})] / \Sigma_{\mathbf{q}} \gamma^{2}(\mathbf{q})$. Calculating the constant with the Fröhlich matrix element $[\gamma(\mathbf{q})$ $\sim 1 / q]$, we find $\gamma=0.57$ in the cubic lattice and $\gamma=0.44$, $\gamma=0.255$ in the cuprate lattice for the apex and in-plane oxygen hole, respectively, in fair agreement with the numerical result.

A lighter mass of the SFP compared with the nondispersive SHP is a generic feature of any dispersive electronphonon interaction. As an example, a short-range interaction with dispersive acoustic phonons $\left[\gamma(\mathbf{q}) \sim 1 / q^{1 / 2}, \omega_{\mathbf{q}} \sim q\right]$ also leads to a lighter polaron in the strong-coupling regime compared with the nondispersive SHP. Actually, Holstein ${ }^{4}$ pointed out in his original paper that the dispersion is a vital ingredient of the theory. If one takes into account the intermolecular interaction in the Holstein model ${ }^{48}$ one can get much lighter polarons in this model as well. ${ }^{48,49}$

\section{POLARON-POLARON INTERACTION AND SCREENING}

Polarons interact not only with phonons, but also with each other. The range of the deformation surrounding (Fröhlich) polarons is quite large (Sec. V), so the polaron deformation fields overlap at finite density. Hence one can worry about the effect of the overlap on their stability. ${ }^{25}$ Actually, the long-range polaron-polaron interaction has been discussed in our original papers ${ }^{16,47,19}$ and books. ${ }^{13}$ Taking into account both the long-range attraction of polarons owing to their lattice deformations and the direct Coulomb repulsion, the residual long-range interaction has been found to be rather weak and repulsive. The Fourier component of the residual polaron-polaron interaction, $v(\mathbf{q})$, comprising the direct Coulomb repulsion and the attraction mediated by phonons, is given by

$$
v(\mathbf{q})=\frac{4 \pi e^{2}}{\epsilon q^{2}}-|\gamma(\mathbf{q})|^{2} \omega_{\mathbf{q}}
$$

In the long-wave limit $(q \ll \pi)$, the Fröhlich interaction dominates in the attractive part, so we have

$$
|\gamma(\mathbf{q})|^{2} \omega=\frac{4 \pi e^{2}\left(\epsilon^{-1}-\epsilon_{0}^{-1}\right)}{q^{2}},
$$

where $\epsilon$ and $\epsilon_{0}$ are the high-frequency and static dielectric constants of the host ionic insulator. Hence, at large distances the polaron-polaron interaction is repulsive:

$$
v_{i j}=\frac{e^{2}}{\epsilon_{0}|\mathbf{m}-\mathbf{n}|} .
$$

Optical phonons nearly nullify the bare Coulomb repulsion in ionic solids if $\epsilon_{0} \gg 1$, which is normally the case in oxides. Hence there is no effect of the overlapping deformations on the small polaron stability.

In the absence of bipolarons (see below), one can apply the canonical random phase approximation to calculate the dielectric response function of polarons:

$$
\epsilon(\mathbf{q}, \Omega)=1-2 v(\mathbf{q}) \sum_{\mathbf{k}} \frac{n_{\mathbf{k}+\mathbf{q}}-n_{\mathbf{k}}}{\Omega-\epsilon_{\mathbf{k}}+\epsilon_{\mathbf{k}+\mathbf{q}}}
$$

This expression describes the response of small polarons to a perturbation of a frequency $\Omega \leqslant \omega$, when phonons in the polaronic cloud are not excited. In the static limit at large distances (or $q \rightarrow 0$ ), we obtain the usual Debye screening with a rather small Debye radius owing to a heavy mass. Actually, for a temperature larger than the polaronic half-bandwidth, one can expand the polaron distribution function as

$$
n_{\mathbf{k}} \simeq \frac{n}{2}\left(1-\frac{(2-n) \epsilon_{\mathbf{k}}}{2 T}\right),
$$

with $n$ the density of polarons, to get

$$
\epsilon(q, 0)=1+\frac{q_{s}^{2}}{q^{2}},
$$




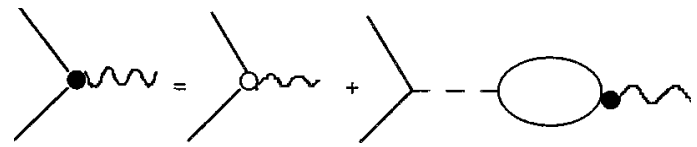

FIG. 8. Electron-phonon vertex (dark circle) screened by the Coulomb interaction (dashed line).

where $q_{s}=\left[2 \pi e^{2} n(2-n) / T \epsilon_{0}\right]^{1 / 2}$. However, already for a finite but rather low-frequency $\Omega \geqslant w$ the polaron response becomes dynamic,

$$
\epsilon(\mathbf{q}, \Omega)=1-\frac{\omega_{p}^{2}(\mathbf{q})}{\Omega^{2}},
$$

with the temperature-dependent polaron plasma frequency

$$
\omega_{p}^{2}(\mathbf{q})=2 v(\mathbf{q}) \sum_{\mathbf{k}} n_{\mathbf{k}}\left(\epsilon_{\mathbf{k}+\mathbf{q}}-\epsilon_{\mathbf{k}}\right),
$$

proportional to the inverse temperature at $T \gg w$.

Considering the electron-phonon interaction in a multipolaron system, one has to take into account the dynamic properties of the response function. One can believe $\mathrm{e}^{24,25}$ that the long-range Fröhlich interaction becomes short range (Holstein) due to screening. This is not true. Replacing the bare electron-phonon interaction $\gamma(\mathbf{q})$ by a screened one $\gamma_{\mathrm{sc}}(\mathbf{q}, \omega)$ as shown in Fig. 8, we obtain

$$
\gamma_{\mathrm{sc}}(\mathbf{q}, \omega)=\frac{\gamma(\mathbf{q})}{\epsilon(\mathbf{q}, \omega)} .
$$

In the long-wave limit the response of polarons at the optical phonon frequency is dynamic, because $\omega \gg q v$ ( $v$ is the characteristic group velocity of polarons). Also, their (renormalized) plasma frequency $\omega_{p}(\mathbf{q})$ is lower than the optical phonon frequency due to the large static dielectric constant, enhanced effective mass, and relatively low density of polarons. Therefore, the singular behavior of $\gamma(\mathbf{q}) \sim 1 / q$ is unaffected by the screening. The optical phonon frequency remains almost unchanged as well. ${ }^{52}$ Polarons are slow enough and cannot screen the high-frequency crystal field oscillations. As a result, the interaction with the high-frequency optical phonons in ionic polaron solids remains long range. Chakraverty et al..$^{24,25}$ failed to understand that the mobility of carriers determines the screening rather than their number.

Another important point is the possibility of the Wigner crystallization of the (bi)polaronic liquid. ${ }^{13}$ Because the residual long-range repulsion is relatively weak, the relevant dimensionless parameter $r_{s}=m^{*} e^{2} / \epsilon_{0}(4 \pi n / 3)^{1 / 3}$ is not very large in doped cuprates. Wigner crystallization appears around $r_{s} \simeq 100$ or larger, which corresponds to the atomic density of polarons, $n \leqslant 10^{-6}$, with $\epsilon_{0}=30$ and $m^{*}=5 m_{e}$. This estimate tells us that the carriers in superconducting cuprates are in a liquid state.

\section{BIPOLARON CONDENSATION AND SUPERCONDUCTING $T_{c}$ IN CUPRATES}

The Fröhlich interaction together with a short-range deformation potential can easily overcome the Coulomb repulsion at a distance about the lattice constant. Then (owing to a narrow band) polarons form real space small bipolarons

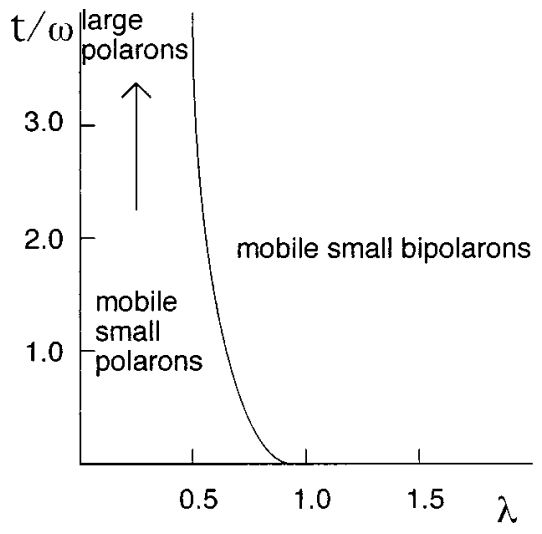

FIG. 9. " $t / \omega$ - $\lambda$ phase" diagram with a small-bipolaron (BoseEinstein condensation) domain, a large-polaron (BCS) domain, and a region of unbound small polarons for $z=6, \gamma=0.4$, and Coulomb pseudopotential $\mu=0.5$.

rather than the Cooper pairs. We can estimate the characteristic parameters $\lambda$ and $t / \omega$ of the bipolaronic instability. The characteristic attractive potential is $V=z t(\lambda-\mu)$, where $\mu$ is the dimensionless Coulomb pseudopotential. A bound state of two polarons appears if ${ }^{53}$

$$
V \geqslant \frac{\pi^{2}}{8 m^{*}} \text {. }
$$

Substituting the polaron mass $m^{*}=\exp (\gamma \lambda z t / \omega) / 2 t$, we find

$$
\frac{t}{\omega} \geqslant(\gamma z \lambda)^{-1} \ln \left[\frac{\pi^{2}}{4 z(\lambda-\mu)}\right] .
$$

The corresponding "phase" diagram is shown in Fig. 9. Bipolarons are formed about at $\lambda \geqslant \mu+\pi^{2} / 4 z$ in the nonadiabatic and intermediate regime $t / \omega \simeq 1$. In the case of the Fröhlich interaction, there is no sharp transition between small and large polarons as one can see in Fig. 7. However, due to the fact that the Lang-Firsov transformation is practically exact in the whole region of coupling for the nonadiabatic and intermediate regime (up to $t / \omega=2$ ), the carriers are small polarons independent of the value of $\lambda$ in this regime. It means that the radius of their wave function is about atomic size and they tunnel together with the entire phonon cloud no matter how "thin" the cloud is. Our estimates are fully confirmed by the numerical simulations of ionic perovskite lattices ${ }^{31}$ which established the existence of stable intersite bipolarons in doped cuprates.

In contrast with BCS theory, the bipolaron theory allows us $^{54,55}$ to "integrate out" the interaction and express $T_{c}$ via the static response functions. In the framework of BCS theory (largely independent of the nature of coupling), the critical temperature is fairly well approximated by McMillan's formula (see Ref. 56),

$$
T_{c}=\frac{\omega}{1.45} \exp \left[-\frac{1.04(1+\lambda)}{\lambda-\mu_{c}^{*}(1+0.62 \lambda)}\right],
$$

which works well for simple metals and their alloys. There are no general restrictions on the BCS value of $T_{c}$ if the dielectric function formalism is properly applied. ${ }^{57}$ Allen and Dynes $^{58}$ found that in the strong-coupling limit $\lambda \gg 1$ the critical temperature might be as high as $T_{c} \simeq \omega \lambda^{1 / 2} / 2 \pi$. Nev- 
ertheless, applying this kind of theory to high- $T_{c}$ cuprates is problematic. Since the bare electron bands are narrow, strong correlations are important, giving rise to a doped Mott insulator. As a result, the Coulomb pseudopotential and $\lambda$ are ill defined and polaronic effects are important as in many doped semiconductors. ${ }^{13}$ Taking the "magic" numbers $\lambda=0.5$, $\mu^{*}=0.14$ and the experimental Debye temperature $\omega$ $=400 \mathrm{~K}$ one obtains $T_{c} \simeq 2 \mathrm{~K}$ with Eq. (44)—clearly too low to explain the high $T_{c}$. One could hardly expect that the Coulomb pseudopotential is lower than 0.1 because the Tolmachev-Morel-Anderson logarithm cannot be large in narrow bands. In fact, $\mu^{*}$ is of the order of the bare Coulomb repulsion, $\mu^{*} \simeq \mu \simeq 1$. Hence, an estimate of $T_{c}$ in cuprates within BCS theory appears to be an exercise in calculating $\mu^{*}$ rather than $T_{c}$ itself. Nor can one increase $\lambda$ without accounting for a polaron collapse of the band and bipolaron formation. As discussed above, this appears at $\lambda \simeq 0.5$ for uncorrelated polarons, Fig. 9, and even for a smaller value of the bare electron-phonon coupling in strongly correlated models. ${ }^{28,29}$ Of course, one can argue ${ }^{59}$ that a renormalized value of the coupling $\tilde{\lambda} \sim \lambda /(1-2 \lambda)$ appears in Eq. (44), rather than a bare $\lambda$ because of the familiar Migdal's softening of the phonon spectrum. That leaves some space for high $T_{c}$ in the region of the applicability of the Eliashberg theory (i.e., $\lambda \leqslant 0.5$ ) where nonadiabatic (vertex) corrections may play a role. ${ }^{60,61}$ The final answer rests with experiment, which has already shown a non-Fermi-liquid normal and a non-BCS superconducting state of doped cuprates (see, for example, Ref. 13). As has been experimentally established, ${ }^{62}$ there is a strong coupling of carriers with $c$-axis-polarized optical phonons in high- $T_{c}$ cuprates $(\omega \simeq 75 \mathrm{meV}$, which is about $t / 2$ in $\mathrm{YBa}_{2} \mathrm{Cu}_{3} \mathrm{O}_{6+x}$ ). Due to a low $c$-axis conductivity and a high phonon frequency, this coupling is not screened representing a long-range Fröhlich interaction with $\lambda \simeq 1,{ }^{63}$ which provides mobile small (bi)polarons. ${ }^{47}$

In the framework of bipolaron theory, the critical temperature is determined by the bipolaron energy spectrum. Quite generally, the bipolaron energy spectrum is a degenerate doublet due to two ( $x$ and $y$ ) oxygen orbitals elongated along the $\mathrm{CuO}_{2}$ planes. ${ }^{47}$ The energy band minima are found at the Brillouin zone boundary $( \pm \pi, 0)$ and $(0, \pm \pi)$ rather than at the $\Gamma$ point owing to the opposite sign of the $p p \sigma$ and $p p \pi$ oxygen hopping integrals. Near these points the effective mass approximation is applied with the following result for the $x$ and $y$ bipolaron bands: ${ }^{47}$

$$
E_{\mathbf{k}}^{x, y}=\frac{\hbar^{2} k_{x, y}^{2}}{2 m_{x}}+\frac{\hbar^{2} k_{y, x}^{2}}{2 m_{y}}+2 t_{\perp}\left[1-\cos \left(k_{z} d\right)\right],
$$

where $k_{x, y}$ are taken relative $( \pm \pi, 0)[$ or $(0, \pm \pi)]$ points, $d$ is the interplane distance, and $t_{\perp}$ is the interplane bipolaron hopping integral. The bipolaron is about 4 times heavier than the SFP. Two different bands are not mixed because the nearest-neighbor hopping integrals between $x$ and $y p$ orbitals are zero. While each of them is not invariant under crystal symmetry, the degenerate doublet represents an irreducible representation. Under a $\pi / 2$ rotation the $x$ band transforms into $y$ and vice versa.

The condensation temperature of these bipolarons has been recently calculated and compared with the experiment. ${ }^{54,55}$ Substituting the spectrum, Eq. (45), into the density sum rule,

$$
\sum_{\mathbf{k}, i=(x, y)}\left[\exp \left(E_{\mathbf{k}}^{i} / T_{c}\right)-1\right]^{-1}=n_{B},
$$

one readily obtains $T_{c}$ as

$$
T_{c}=f \frac{3.31\left(n_{B} / 2\right)^{2 / 3}}{\left(m_{x} m_{y} m_{c}\right)^{1 / 3}},
$$

where the coefficient $f$ is almost unity in a wide range of the anisotropy $t_{\perp} / T_{c},{ }^{55}$ and $m_{c}=1 / 2\left|t_{\perp}\right| d^{2}$. This expression is rather ambiguous so far because the effective mass tensor as well as the bipolaron density $n_{B}$ is unknown and doping dependent. Fortunately, one can express the band-structure parameters through the in-plane, $\lambda_{a b}=\left[m_{x} m_{y} / 8 \pi n_{B} e^{2}\left(m_{x}\right.\right.$ $\left.\left.+m_{y}\right)\right]^{1 / 2}$, and out-of-plane $\lambda_{c}=\left[m_{c} / 16 \pi n_{B} e^{2}\right]^{1 / 2}$, penetration depth. The bipolaron density is expressed through the in-plane Hall constant (just above the transition) as 47

$$
R_{H}=\frac{1}{2 e n_{B}} \frac{4 m_{x} m_{y}}{\left(m_{x}+m_{y}\right)^{2}} .
$$

As a result, one obtains

$$
T_{c}=1.64\left(\frac{e R_{H}}{\lambda_{a b}^{4} \lambda_{c}^{2}}\right)^{1 / 3},
$$

with $T_{c}$ measured in kelvin, $e R_{H}$ in $\mathrm{cm}^{3}$, and $\lambda$ in $\mathrm{cm}$. Hence our theory yields a parameter-free expression, which unambiguously tells us how close cuprates are to the BoseEinstein condensation regime. This expression has been compared with the experimental $T_{c}$ of more than 30 different cuprates, for which both $\lambda_{a b}$ and $\lambda_{c}$ are measured along with $R_{H}{ }^{55}$ The theoretical $T_{c}$ coincides with the experimental one within the experimental error bar for the penetration depth (about $\pm 10 \%$ ) no matter what the doping level is. A few examples are $\mathrm{La}_{1.8} \mathrm{Sr}_{0.2} \mathrm{CuO}_{4} \quad\left[\lambda_{a b}=2000 \AA, \quad \lambda_{c}\right.$ $\left.=25400 \AA, R_{H}=0.8 \times 10^{-3}\left(1 / \mathrm{C} \mathrm{cm}^{3}\right)\right], T_{c}^{\exp }=36.2 \mathrm{~K}$, and our theoretical value, Eq. (49), is $T_{c}=38 \mathrm{~K}$; $\mathrm{YBa}_{2} \mathrm{Cu}_{3} \mathrm{O}_{7}$ $\left[\lambda_{a b}=1400 \AA, \quad \lambda_{c}=12600 \AA, \quad R_{H}=1.2 \times 10^{-3}\left(1 / \mathrm{C} \mathrm{cm}^{3}\right)\right]$, $T_{c}^{\exp }=92.5 \mathrm{~K}$, and the theoretical value is $T_{c}=111 \mathrm{~K}$; $\mathrm{YBa}_{2} \mathrm{Cu}_{3} \mathrm{O}_{6.84} \quad\left[\lambda_{a b}=1771 \AA, \quad \lambda_{c}=15570 \AA, \quad R_{H}=1.9\right.$ $\left.\times 10^{-3}\left(1 / \mathrm{C} \mathrm{cm}^{3}\right)\right], T_{c}^{\exp }=83.7 \mathrm{~K}$, and the theoretical value is $T_{c}=83 \mathrm{~K}$.

One can argue that cuprates belong to a two-dimensional (2D) $X Y$ universality class with the Kosterlitz-Thouless critical temperature $T_{\mathrm{KT}}$ due to a large anisotropy. ${ }^{64-66}$ If this is the case, then one could not discriminate the Cooper pairs with respect to bipolarons. The Kosterlitz-Thouless temperature, expressed through the in-plane penetration depth, is ${ }^{66}$

$$
T_{\mathrm{KT}} \simeq \frac{0.9 d}{16 \pi e^{2} \lambda_{a b}^{2}} .
$$

It appears significantly (about twice) higher than the experimental value in most cases. Also, many cuprates do not share the critical behavior of the BCS superfluids or the universal (3D) $x-y$ properties of neutral superfluids like ${ }^{4} \mathrm{He},{ }^{67,68}$ but exhibit the critical behavior of charged bosons. These observations favor the 3D Bose-Einstein condensation of charged 
bosons as the mechanism of high $T_{c}$ rather than any lowdimensional phase-fluctuation scenario. The fascinating experimental results by Franck, ${ }^{69}$ Zhao et al. ${ }^{70}$ and Zhao et al. ${ }^{71}$ who measured the oxygen isotope effect on the critical temperature and the carrier mass in many oxides, lead to the conclusion that charged bosons are bipolarons.

The size of the intersite bipolaron has been well established in numerical studies by Catlow et al. ${ }^{31}$ This bipolaron occupies a volume, which is 5 times smaller than the unit cell volume in $\mathrm{YBa}_{2} \mathrm{Cu}_{3} \mathrm{O}_{7}$. There is about one bipolaron per unit (chemical) cell, which includes 13 ions. As a result, there is no overcrowding problem, and hole carriers can be treated as charged bosons. Nevertheless, the boson-boson interaction might lead to self-energy effects and to some renormalization of the effective mass tensor. It is important that Eq. (49) does not contain the mass and, hence, is not affected by the interaction. The theoretical value of the $a b$ bipolaron mass, $\simeq(10-20) m_{e}$ [i.e., about $(3-5) m_{e}$ for a polaron, Fig. $7]$, fits well the experimental values of $\lambda_{a b}$. The careful exploration of the discrete nondispersive Holstein-Hubbard model by La Magna and Pucci ${ }^{35}$ and more recently by Bonca, Katrasnik, and Trugman ${ }^{72}$ showed that due to exchange and nonadiabaticity effects an intersite bipolaron with a relatively small effective mass is stable and mobile even in this model, which is generally unfavorable for tunneling.

It becomes clear that bipolaron theory describes remarkably well the experimental critical temperature and the London penetration depth of superconducting cuprates with no parameters to fit contrary to conclusions by Chakraverty et al. ${ }^{24}$ which originate in the use of an incorrect bipolaron energy spectrum. ${ }^{54}$ In particular, Ref. 24 used our nonadiabatic expression for the mass of the on-site bipolaron with adiabatic parameters. As we have shown in Sec. IV, this results in an overestimation of the mass by a few orders of magnitude, Fig. 3(b). They also used our expression for the intersite bipolaron hopping by leaving out the numerical coefficient $\gamma$ which lowers the (bi)polaron mass by about two orders of magnitude as discussed in Sec. V. Their conclusion that the Holstein model represents well the electron-phonon interaction in ionic polaronic solids is disputed. The Fröhlich interaction cannot be reduced to a short-range one in the multipolaron system (Sec. VI). As we have shown above, (bi)polarons exist in the Bloch states at low temperatures, and the bipolaronic liquid cannot be crystallized at any relevant level of doping. The correct phase diagram of electrons, coupled with phonons (see Ref. 15 and Fig. 9), includes the BCS ground state in the weak-coupling regime and high- $T_{c}$ bipolaronic superconductor for the strong coupling contrary to the diagram by Chakraverty, ${ }^{73}$ where (bi)polarons are completely localized.

\section{COHERENT AND INCOHERENT SPECTRAL WEIGHT AND POLARONIC ARPES}

A number of thermodynamic, magnetic and kinetic properties of cuprates have been understood in the framework of the bipolaron theory (for a review, see Refs. 13, 10, and 19). On the other hand, the single-particle spectral function seen by angle-resolved photoemission spectroscopy (ARPES) (Ref. 74) was interpreted by several authors as a Fermi-liquid feature of the normal state incompatible with bipolarons. Most (but not all) of these measurements produced a large Fermi surface. This should evolve with doping as $(1-x)$ in a clear contradiction with low-frequency kinetics and thermodynamics, which show an evolution proportional to $x$ ( $x$ is the number of holes introduced by doping). Recently, it has been established, however, that there is a normal state gap in ARPES and superconducting-insulating-normal (SIN) tunneling, existing well above $T_{c}$ irrespective of the doping level. ${ }^{7-76}$ The "Fermi surface" showed missing segments. A plausible explanation is that there are two liquids in the cuprates, the normal Fermi liquid and the charged Bose liquid, as suggested by several independent experiments ${ }^{77-80}$ (this mixture was theoretically discussed a while ago ${ }^{81}$ ).

The single-particle spectral function of a bipolaronic system has been recently derived. ${ }^{82,19}$ It describes the spectral features of tunneling and photoemission in cuprates, in particular, the temperature-independent gap and the anomalous $\mathrm{gap} / T_{c}$ ratio, injection and emission asymmetry both in magnitude and shape, zero-bias conductance at zero temperature, the spectral shape inside and outside the gap region, temperature and doping dependence and dip-hump structure of the tunneling conductance, and photoemission. In the following we briefly analyze some essential SHP and SFP spectral features.

Bipolarons pin the chemical potential inside the charge transfer gap, half the bipolaron binding energy above the oxygen band edge. This binding energy as well as the singlet-triplet bipolaron exchange energy is thought to be the origin of the normal-state pseudogaps, as first proposed by us in Ref. 83. In overdoped samples the bipolaron and polaron bands might overlap because the bipolaron binding energy becomes small, so the chemical potential might enter the oxygen band, as mentioned above. The strong coupling with high-frequency phonons, experimentally established for many oxides, leads to the high-energy spectral features of a single-particle (oxygen hole) spectral function in an energy window about twice the Franck-Condon (polaronic) level shift, $2 E_{p} \sim 0.5-1 \mathrm{eV}$, and to the band-narrowing effect. All major features of the polaronic spectral function can be derived by applying the Lang-Firsov canonical transformation, Eq. (4). With this transformation the hole Matsubara Green's function (GF) is expressed as a convolution of the coherent polaron GF and the multiphonon correlation function $\sigma\left(\mathbf{m}, \Omega_{n}\right),{ }^{19}$

$$
\mathcal{G}\left(\mathbf{k}, \omega_{n}\right)=\frac{T}{N} \sum_{\omega_{n^{\prime}}, \mathbf{m}, \mathbf{k}^{\prime}} \frac{\sigma\left(\mathbf{m}, \omega_{n^{\prime}}-\omega_{n}\right) e^{i\left(\mathbf{k}-\mathbf{k}^{\prime}\right) \cdot \mathbf{m}}}{i \omega_{n^{\prime}}-\xi_{\mathbf{k}^{\prime}}}
$$

where the multiphonon correlation function $\sigma(\mathbf{m}, \tau)$ $=T \Sigma_{n} e^{-i \Omega_{n} \tau} \sigma\left(\mathbf{m}, \Omega_{n}\right)$ is found as

$$
\sigma(\mathbf{m}, \tau)=\exp \left(\frac{1}{2 N} \sum_{\mathbf{q}}|\gamma(\mathbf{q})|^{2} f_{\mathbf{q}}(\mathbf{m}, \tau)\right) .
$$

Here

$$
\begin{aligned}
f_{\mathbf{q}}(\mathbf{m}, \tau)= & {\left[\cos (\mathbf{q} \cdot \mathbf{m}) \cosh \left(\omega_{\mathbf{q}}|\tau|\right)-1\right] \operatorname{coth} \frac{\omega_{q}}{2 T} } \\
& +\cos (\mathbf{q} \cdot \mathbf{m}) \sinh \left(\omega_{\mathbf{q}}|\tau|\right),
\end{aligned}
$$


with $\mathbf{m}$ the lattice vector, $\omega_{n}=\pi T(2 n+1), \quad n=0, \pm 1$, $\pm 2 \ldots$, and $\Omega_{n}=2 \pi T n$.

In the case of dispersionless phonons and the short-range (Holstein) interaction with a $q$-independent matrix element $\left[\omega_{\mathbf{q}}=\omega,|\gamma(\mathbf{q})|^{2}=2 g^{2}\right]$, one can readily calculate the Fourier component of $\sigma(\mathbf{m}, \tau)$ to obtain ${ }^{19}$

$$
\begin{aligned}
\mathcal{G}\left(\mathbf{k}, \omega_{n}\right)= & \frac{Z}{i \omega_{n}-\xi_{\mathbf{k}}}+\frac{Z}{N} \sum_{l=1}^{\infty} \frac{g^{2 l}}{l !} \\
& \times \sum_{\mathbf{k}^{\prime}}\left(\frac{n_{\mathbf{k}^{\prime}}}{i \omega_{n}-\xi_{\mathbf{k}^{\prime}}+l \omega}+\frac{1-n_{\mathbf{k}^{\prime}}}{i \omega_{n}-\xi_{\mathbf{k}^{\prime}}-l \omega}\right) .
\end{aligned}
$$

The Green function of a polaronic carrier comprises two different contributions. The first coherent k-dependent term arises from the polaron band tunneling. The spectral weight of the coherent part is strongly (exponentially) suppressed as $Z=\exp \left(-g^{2}\right)$, in agreement with cluster numerical studies, ${ }^{14,84}$ and the effective mass is strongly enhanced, $\xi_{\mathbf{k}}$ $=Z E_{\mathbf{k}}-\mu$ (we include the polaronic level shift into the chemical potential, $\mu)$. Here $E(\mathbf{k})$ is the bare [local density approximation (LDA)] hole band dispersion in a rigid lattice. The second $\mathbf{k}$-independent contribution describes the excitations accompanied by the emission and absorption of phonons. We believe that this term $I_{\text {incoh }}(E)$ is responsible for the asymmetric background in the optical conductivity and in the photoemission spectra of cuprates and manganites. Its spectral density spreads over a wide energy range of about twice the polaron level shift, $E_{p}=g^{2} \omega$. On the contrary, the coherent term shows an angular dependence in the energy range of the order of the polaron bandwidth $2 w$ $\equiv Z D$, where $D \simeq 2 z t$ is the bare (LDA) bandwidth.

It is remarkable that for any finite-radius interaction with a $q$-dependent matrix element the coherent part of the GF takes the same form as Eq. (53), but with a different spectral weight $(Z)$ and effective mass $\left(Z^{\prime}\right)$ renormalization exponents. ${ }^{85}$ Also, some $\mathbf{k}$ dependence of the incoherent background, $I_{\text {incoh }}(\mathbf{k}, E)$, appears if the matrix element of the electron-phonon interaction depends on $q .{ }^{86}$ Hence, in general, the polaron spectral function is given by

$$
A(\mathbf{k}, E) \propto Z \delta\left(E+\xi_{\mathbf{k}}\right)+I_{\text {incoh }}(\mathbf{k}, E),
$$

with the same $Z=\exp \left(-E_{p} / \omega\right)$ as in the case of the Holstein polaron, but with the SFP bandwidth much less reduced, $\xi_{\mathbf{k}}$ $=Z^{\prime} E(\mathbf{k})-\mu$, where $Z^{\prime}=\exp \left(-\gamma E_{p} / \omega\right)$. These SFP spectral features could explain the apparent discrepancy between a small coherent spectral weight and a relatively moderate mass enhancement, $m^{*} \sim 3 m_{e}-10 m_{e}$ (depending on doping), of carriers in oxides, as measured optically and thermodynamically, respectively. ${ }^{87,88}$ It is important to emphasize that the small coherent weight $Z$ in Eq. (53) does not affect the thermodynamic (or low-frequency) response of polarons. This response depends on $Z^{\prime}$ in the polaron kinetic energy, $2 w=Z^{\prime} D$, as discussed in Sec. V. Compared with the Holstein polaron, the maximum of the infrared optical conductivity of the SFP is shifted to lower frequencies of the order of $2 \gamma E_{p},{ }^{8,12}$ in agreement with those optical experiments, ${ }^{89}$ which distinguish between incoherent and Drude contributions. The low-energy spectral function also depends on the low-frequency thermal lattice, spin, and random fluctuations. The latter can be described as the "Gaussian white noise." As a result, one can quantitatively describe the experimental ARPES in a few cuprates. ${ }^{90}$ The approach is clearly compatible with the doping evolution of thermodynamic and kinetic properties because holes introduced by doping into the oxygen band are the only carriers in the theory. Moreover, the bipolaron energy dispersion with the minima at the Brillouin zone boundaries, Eq. (45), provides a $d$-wave symmetry of the Bose-Einstein condensate in cuprates ${ }^{82}$ as observed in phase-sensitive experiments. ${ }^{91}$

\section{CONCLUSIONS}

Based on the well-established 1/ $\lambda$ perturbation theory, exact cluster diagonalization, and quantum Monte Carlo calculations, I conclude that small polarons and small bipolarons are itinerant quasiparticles existing in the Bloch states at temperatures below the characteristic phonon frequency for any strength of the electron-phonon coupling. This result was analytically established a long time ago. ${ }^{4,5,8,39,15}$ There are a few additional results which can help to resolve some confusion in the polaron literature.

(i) Numerically calculated correlation functions of the two-site Holstein model agree perfectly well with the analytical results based on the Lang-Firsov transformation and $1 / \lambda$ perturbation expansion (Sec. IV).

(ii) The long-range Fröhlich interaction leads to relatively light small polarons with atomic size of the wave function and a large size of the phonon cloud in all dimensions. The effective mass of this polaron is smaller by a few orders of magnitude than the mass of the nondispersive Holstein model in the strong-coupling region. At a weak coupling the Fröhlich polaron is heavier than the Holstein polaron with the same binding energy. These SFP features have been recently found in Ref. 43. Here I have generalized these results for the isotropic three-dimensional Fröhlich interaction underlying the fact that the first-order $1 / \lambda$ expansion is perfectly accurate even in the adiabatic regime $\omega / t \leqslant 1$ for any coupling strength in the case of the Fröhlich interaction (Sec. V).

(iii) Screening in the multipolaron system has been analyzed to show that polarons cannot screen the highfrequency crystal field oscillations because they are slow enough. As a result, the interaction with the high-frequency optical phonons in ionic polaron solids remains long ranged (Sec. VI).

(iv) At large distances the (bi)polaron-(bi)polaron interaction is shown to be repulsive and weak. Optical phonons nearly nullify the bare Coulomb repulsion in ionic solids if $\epsilon_{0} \gg 1$, which is normally the case in oxides. Hence there is no effect of the overlapping deformations on the small polaron stability. If small bipolarons are formed, they cannot be crystallized in the range of parameters typical for cuprates (Sec. VII).

(v) Small mobile bipolarons are formed at the moderate coupling constant $\lambda \geqslant 0.5$, almost independent of the adiabatic ratio (Fig. 9).

(vi) Bipolaron theory provides a parameter-free expression for $T_{c}$, describing $T_{c}$ of many cuprates without any fitting parameters, as has been shown recently. ${ }^{54,55}$ Here I 
argue that the small Fröhlich polaron has the spectral properties compatible with the single-particle tunneling and ARPES measurements in cuprates. The important finding is that the coherent part of its Green's function has a spectral weight $Z$ different from the bandwidth renormalization exponent $Z^{\prime}$ with $Z^{\prime} \gg Z$. This SFP spectral feature can explain the apparent discrepancy between a small coherent spectral weight and a relatively moderate mass enhancement in $\mathrm{cu}-$ prates and manganites.

The objections ${ }^{21,22,24,25}$ recently raised against HolsteinLang-Firsov polaron theory and our theory of bipolaronic superconductivity are shown (see also Refs. 26 and 44) to be the result of an erroneous definition of the polaron kinetic energy, ${ }^{21}$ an incorrect interpretation of the dynamic correlation functions of the Holstein model,,$^{21,22,45}$ and a misuse ${ }^{24,25}$ of our expressions for the bipolaron effective mass.

\section{ACKNOWLEDGMENTS}

The author greatly appreciates stimulating discussions with P. B. Allen, A. R. Bishop, A. M. Bratkovsky, A. Bussmann-Holder, E. Dagotto, J. T. Devreese, D. M. Eagles, Yu. A. Firsov, L. P. Gor'kov, V. V. Kabanov, P. E. Kornilovitch, E. K. Kudinov, W. Y. Liang, E. G. Maximov, S. von Molnar, J. R. Schrieffer, and G. Zhao.
${ }^{1}$ S. V. Tjablikov, Zh. Eksp. Teor. Fiz. 23, 381 (1952).

${ }^{2}$ J. Yamashita and T. Kurosawa, J. Phys. Chem. Solids 5, 34 (1958).

${ }^{3}$ G. L. Sewell, Philos. Mag. 3, 1361 (1958).

${ }^{4}$ T. Holstein, Ann. Phys. (N.Y.) 8, 325 (1959); 8, 343 (1959).

${ }^{5}$ I. G. Lang and Yu. A. Firsov, Zh. Eksp. Teor. Fiz. 43, 1843 (1962) [Sov. Phys. JETP 16, 1301 (1963)].

${ }^{6}$ E. K. Kudinov and Yu. A. Firsov, Fiz. Tverd. Tela (Leningrad) 7, 546 (1965) [Sov. Phys. Solid State 7, 435 (1965)].

${ }^{7}$ J. Appel, in Solid State Physics, edited by F. Seitz, D. Turnbull, and H. Ehrenreich (Academic, New York, 1968), Vol. 21.

${ }^{8}$ Polarons, edited by Yu. A. Firsov (Nauka, Moscow, 1975).

${ }^{9}$ A. S. Alexandrov and N. F. Mott, Rep. Prog. Phys. 57, 1197 (1994).

${ }^{10}$ J. T. Devreese, in Encyclopedia of Applied Physics, edited by G. L. Trigg (VCH, Weinheim, 1996), Vol. 14, p. 383.

${ }^{11}$ H. Böttger and V. V. Bryksin, Hopping Conduction in Solids (Academie-Verlag, Berlin, 1985).

${ }^{12}$ G. D. Mahan, Many Particle Physics (Plenum, New York, 1990).

${ }^{13}$ A. S. Alexandrov and N. F. Mott, Polarons and Bipolarons (World Scientific, Singapore, 1995); High Temperature Superconductors and Other Superfluids (Taylor \& Francis, London, 1994).

${ }^{14}$ A. S. Alexandrov, V. V. Kabanov, and D. K. Ray, Phys. Rev. B 49, 9915 (1994).

${ }^{15}$ A. S. Alexandrov, Zh. Fiz. Khim. 57, 2731983 [Russ. J. Phys. Chem. 57, 167 (1983)].

${ }^{16}$ A. S. Alexandrov, Phys. Rev. B 46, 2838 (1992).

${ }^{17}$ V. V. Kabanov and O. Yu. Mashtakov, Phys. Rev. B 47, 6060 (1993).

${ }^{18}$ A. S. Aleksandrov (Alexandrov), Pis'ma Zh. Eksp. Teor. Fiz. 46, 128 (1987) [JETP Lett. 46, 107 (1987)].

${ }^{19}$ A. S. Alexandrov, in Models and Phenomenology for Conventional and High-temperature Superconductivity, Proceedings of the Intenational School of Physics "Enrico Fermi," Course CXXXVI, Varenna, 1998, edited by G. Iadonisi, J. R. Schrieffer, and M. L. Chiofalo (IOS Press, Amsterdam, 1998), p. 309.

${ }^{20}$ A. S. Alexandrov and A. M. Bratkovsky, Phys. Rev. Lett. 82, 141 (1999).

${ }^{21}$ E. V. L. de Mello and J. Ranninger, Phys. Rev. B 55, 14872 (1997).

${ }^{22}$ E. V. L. de Mello and J. Ranninger, Phys. Rev. B 58, 9098 (1998).

${ }^{23}$ P. W. Anderson, Phys. World 9, 16 (1997); N. P. Ong and P. W.
Anderson, Phys. Rev. Lett. 79, 4718 (1997).

${ }^{24}$ B. K. Chakraverty, J. Ranninger, and D. Feinberg, Phys. Rev. Lett. 81, 433 (1998).

${ }^{25}$ B. K. Chakraverty, J. Ranninger, and D. Feinberg, Phys. Rev. Lett. 82, 2621 (1999).

${ }^{26}$ Yu. A. Firsov and E. K. Kudinov, Fiz. Tverd. Tela (St. Petersburg) 39, 2159 (1997) [Phys. Solid State 39, 1930 (1997)].

${ }^{27}$ K. A. Müller, in Anharmonic Properties of High- $T_{c}$ Cuprates, edited by D. Mihailovi'c, G. Ruani, E. Kaldis, and K. A. Müller (World Scientific, Singapore, 1995).

${ }^{28}$ A. R. Bishop and M. Salkola, in Polarons and Bipolarons in High- $T_{c}$ Superconductors and Related Materials, edited by E. K. H. Salje, A. S. Alexandrov, and W. Y. Liang (Cambridge University Press, Cambridge, England, 1995), p. 353.

${ }^{29}$ H. Fehske, H. Roder, G. Wellein, and A. Mistriotis, Phys. Rev. B 51, 16582 (1995).

${ }^{30}$ J. Tempere, V. M. Fomin, and J. T. Devreese, Solid State Commun. 101, 661 (1997).

${ }^{31}$ C. R. A. Catlow, M. S. Islam, and X. Zhang, J. Phys.: Condens. Matter 10, L49 (1998).

${ }^{32}$ P. E. Kornilovich and E. R. Pike, Phys. Rev. B 55, R8634 (1997).

${ }^{33}$ H. Fehske, J. Loos, and G. Wellein, Z. Phys. B: Condens. Matter 104, 619 (1997).

${ }^{34}$ A. H. Romero, D. W. Brown, and K. Lindenberg, J. Chem. Phys. 109, 6504 (1998).

${ }^{35}$ A. La Magna and R. Pucci, Phys. Rev. B 53, 8449 (1996).

${ }^{36}$ E. Jeckelmann and S. R. White, Phys. Rev. B 57, 6376 (1998).

${ }^{37}$ P. E. Kornilovich, Phys. Rev. Lett. 81, 5382 (1998).

${ }^{38}$ P. Benedetti and R. Zeyher, Phys. Rev. B 58, 14320 (1998).

${ }^{39}$ A. A. Gogolin, Phys. Status Solidi B 109, 95 (1982).

${ }^{40}$ A. Kongeter and M. Wagner, J. Chem. Phys. 92, 4003 (1990).

${ }^{41}$ F. Marsiglio, Physica C 244, 21 (1995).

${ }^{42}$ H. deRaedt and Ad. Lagendijk, Phys. Rev. B 27, 6097 (1983).

${ }^{43}$ A. S. Alexandrov and P. E. Kornilovich, Phys. Rev. Lett. 82, 807 (1999).

${ }^{44}$ Yu. A. Firsov, V. V. Kabanov, E. K. Kudinov, and A. S. Alexandrov, Phys. Rev. B 59, 12132 (1999); E. V. L. de Mello and J. Ranninger, Phys. Rev. B 59, 12135 (1999).

${ }^{45}$ E. V. L. de Mello and J. Ranninger, Phys. Rev. B 58, 14625 (E) (1998).

${ }^{46}$ V. V. Kabanov (personal communication).

${ }^{47}$ A. S. Alexandrov, Phys. Rev. B 53, 2863 (1996).

${ }^{48}$ M. Zoli, Int. J. Mod. Phys. B 12, 3146 (1998); and (unpublished).

${ }^{49}$ L. Cruzeiro-Hansson, J. C. Eilbeck, J. L. Marin, and F. M. Russell (unpublished). 
${ }^{50}$ D. M. Eagles, Phys. Rev. 130, 1381 (1963).

${ }^{51}$ A. S. Alexandrov and P. E. Kornilovich (unpublished).

${ }^{52}$ A. S. Alexandrov and J. R. Schrieffer, Phys. Rev. B 56, 13731 (1997).

${ }^{53}$ L. D. Landau and E. M. Lifshitz, Quantum Mechanics, 3rd ed. (Pergamon, New York, 1991).

${ }^{54}$ A. S. Alexandrov, Phys. Rev. Lett. 82, 2620 (1999).

${ }^{55}$ A. S. Alexandrov and V. V. Kabanov, Phys. Rev. B 59, 13628 (1999).

${ }^{56}$ D. J. Scalapino, in Superconductivity, edited by R. D. Parks (Deccer, New York, 1969), Vol. 1.

${ }^{57}$ O. V. Dolgov, D. A. Kirzhnits, and E. G. Maximov, Rev. Mod. Phys. 53, 81 (1981).

${ }^{58}$ P. B. Allen and R. C. Dynes, Phys. Rev. B 12, 905 (1975).

${ }^{59}$ E. G. Maximov (personal communication).

${ }^{60}$ P. B. Allen and B. Mitrovic, in Solid State Physics, edited by H. Ehrenreich, F. Seitz, and D. Turnbull (Acadenic, New York, 1982), Vol. 37, p. 1.

${ }^{61}$ C. Grimaldi, L. Pietronero, and S. Strassler, Phys. Rev. Lett. 75, 1158 (1995).

${ }^{62}$ T. Timusk, C. C. Homes, and W. Reichardt, in Anharmonic Properties of High- $T_{c}$ Cuprates, edited by D. Mihailovic, G. Ruani, E. Kaldis, and K. A. Müller (World Scientific, Singapore, 1995), p. 171.

${ }^{63}$ Characteristic values of the polaron coupling constants in oxides can be found in D. M. Eagles, J. Phys. Chem. Solids 25, 1243 (1964).

${ }^{64}$ B. J. Alder and D. S. Peters, Europhys. Lett. 10, 1 (1989).

${ }^{65}$ V. L. Pokrovsky, Pis'ma Zh. Eksp. Teor. Fiz. 47, 539 (1988) [JETP Lett. 47, 629 (1988)].

${ }^{66}$ V. J. Emery and S. A. Kivelson, Nature (London) 374, 434 (1995).

${ }^{67}$ A. Junod, in Studies of High Temperature Superconductors, edited by A. Narlikar (Nova Science, Commack, NY, 1996), Vol. 19, p. 1; A. Junod (unpublished).

${ }^{68}$ A. S. Alexandrov, W. H. Beere, V. V. Kabanov, and W. Y. Liang, Phys. Rev. Lett. 79, 1551 (1997).

${ }^{69}$ J. P. Franck, in Physical Properties of High Temperature Superconductors IV, edited by D. M. Ginsberg (World Scientific, Singapore, 1994), p. 189.

${ }^{70}$ G. Zhao, K. K. Singh, and D. E. Morris, Phys. Rev. B 50, 4112 (1994).
${ }^{71}$ G. Zhao, M. B. Hunt, H. Keller, and K. A. Müller, Nature (London) 385, 236 (1997).

${ }^{72}$ J. Bonca, T. Katrasnik, and S. A. Trugman (unpublished).

${ }^{73}$ B. K. Chakraverty, J. Phys. (France) Lett. 40, L-99 (1979).

${ }^{74}$ Z.-X. Shen and J. R. Schrieffer, Phys. Rev. Lett. 78, 1771 (1997) and references therein.

${ }^{75}$ N. L. Saini, A. Bianeoni, A. Lanzara, J. Avila, M. C. Asensio, S. Tajima, G. D. Gu, and N. Koshizuka, Phys. Rev. Lett. 82, 2619 (1998).

${ }^{76}$ Ch. Renner, B. Revaz, J. Y. Genoud, K. Kadowaki, and Ø. Fischer, Phys. Rev. Lett. 80, 149 (1998).

${ }^{77}$ K. A. Müller, G. M. Zhao, K. Conder, and H. Keller, J. Phys.: Condens. Matter 10, L291 (1998).

${ }^{78}$ D. Mihailovi'c, C. Stevens, B. Podobnik, D. C. Smith, and J. F. Ryan, J. Supercond. 10, 337 (1997).

${ }^{79}$ J. Halbritter, Physica C 302, 221 (1998).

${ }^{80}$ J. Hofer, J. Karpinski, M. Willemin, G. I. Meijer, E. M. Kopnin, R. Molinski, C. Rossel, and H. Keller, Physica C 297, 103 (1998).

${ }^{81}$ A. S. Aleksandrov (Alexandrov) and A. B. Khmelinin, Fiz. Tverd. Tela (Leningrad) 28, 3403 (1986) [Sov. Phys. Solid State 28, 1915 (1986)]. We have proved that the hybridization of bosons and fermions does not play any role and cannot lead to high $T_{c}$ [see A. S. Alexandrov, J. Phys.: Condens. Matter 8, 6923 (1996); Physica C 274, 237 (1997); 316, 239 (1999)].

${ }^{82}$ A. S. Alexandrov, Physica C 305, 46 (1998).

${ }^{83}$ A. S. Alexandrov, Physica C 182, 327 (1991).

${ }^{84}$ C. Zhang, E. Jeckelmann, and S. R. White, cond-mat/9812353 (unpublished).

${ }^{85}$ A. S. Alexandrov and C. Sricheewin (unpublished).

${ }^{86}$ G. J. Kaye, Phys. Rev. B 57, 8759 (1998).

${ }^{87}$ Y. Okimoto, T. Katsufuji, T. Ishikawa, A. Urushibara, T. Arima, and Y. Tokua, Phys. Rev. Lett. 75, 109 (1995).

${ }^{88}$ D. S. Dessau, T. Saiton, C. H. Park, Z. X. Shen, P. Villella, N. Hamada, Y. Moritomo, and Y. Tokura, Phys. Rev. Lett. 81, 192 (1998); cond-mat/9904050 (unpublished).

${ }^{89}$ D. Mihailovi'c, C. M. Foster, K. Voss, and A. J. Heeger, Phys. Rev. B 42, 7989 (1990).

${ }^{90}$ A. S. Alexandrov and C. J. Dent, Phys. Rev. B 60, 15414 (1999).

${ }^{91}$ For a review, see J. Annett, N. Goldenfeld, and A. J. Legget, in Physical Properties of High Temperature Superconductors, edited by D. M. Ginsberg (World Scientific, Singapore, 1996), Vol. 5, p. 375 . 